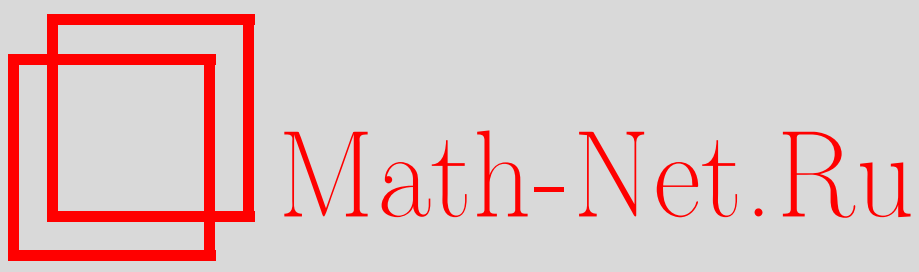

A. P. Pozhidaev, On endomorphs of right-symmetric algebras, Sibirsk. Mat. Zh., 2020, Volume 61, Number 5, 1077-1086

DOI: https://doi.org/10.33048/smzh.2020.61.509

Use of the all-Russian mathematical portal Math-Net.Ru implies that you have read and agreed to these terms of use http://www . mathnet.ru/eng/agreement

Download details:

IP: 54.224 .187 .69

April 26, 2023, 08:03:54

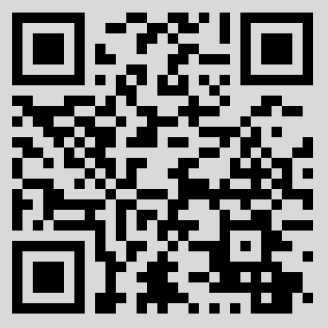


Сибирский математический журнал Сентябрь-октябрь, 2020. Том 61, № 5

УДК 512.57

\title{
ОБ ЭНДОМОРФАХ ПРАВОСИММЕТРИЧЕСКИХ АЛГЕБР
}

А. П. Пожидаев

\begin{abstract}
Аннотация. Вводится понятие эндоморфа $E(\mathscr{A})$ (супер)алгебры $\mathscr{A}$ и доказывается, что $E(\mathscr{A})$ является простой (супер)алгеброй, если $\mathscr{A}$ - это не алгебра скалярного умножения. Если $\mathscr{A}$ - правосимметрическая супералгебра, то и $E(\mathscr{A})$ - правосимметрическая супералгебра. Тем самым строится широкий класс простых (правосимметрических) (супер)алгебр, содержащих матричную подалгебру с общей единицей. Находятся алгебра дифференцирований эндоморфа унитальной алгебры $\mathscr{A}$ и группа автоморфизмов простой правосимметрической алгебры $E\left(V_{n}\right)$ (эндоморфа прямой суммы полей).
\end{abstract}

DOI 10.33048/smzh.2020.61.509

Ключевые слова: эндоморф, правосимметрическая алгебра, левосимметрическая алгебра, простая алгебра, дифференцирование, автоморфизм, прелиева алгебpa.

\section{Введение}

Алгебра $\mathscr{A}$ называется правосимметрической, если ассоциатор на $\mathscr{A}$ правосимметричен, т. е. он симметричен относительно двух последних аргументов:

$$
(x, y, z)=(x, z, y)
$$

для всех $x, y, z \in \mathscr{A}$, где $(x, y, z):=(x y) z-x(y z)-$ accouиатор $x, y, z$ (символ $:=$ означает равенство по определению). В операторном виде (1) можно записать так:

$$
\left[R_{y}, R_{z}\right]=R_{[y, z]}
$$

где $[y, z]:=y z-z y-$ коммутатор элементов $y$ и $z$, а $R_{y}$ - оператор правого умножения на $y \in \mathscr{A}: x R_{y}=x y$ для всех $x \in \mathscr{A}$.

Правосимметрические алгебры являются важным обобщением ассоциативных алгебр. Эти алгебры Ли-допустимы, и они естественно возникают в разных областях. Их антиизоморфный аналог - левосимметрические алгебры. Повидимому, левосимметрические алгебры были впервые введены Кэли в 1857 г. В 1961 г. Кожуль использовал их при изучении действий аффинных преобразований [1]. В 1963 г. Винберг применил левосимметрические алгебры для классификации выпуклых однородных конусов [2], а Герстенхабер использовал их при изучении деформаций алгебр [3]. K настоящему времени левосимметрическим алгебрам (также известным под другими именами, например, как прелиевы алгебры) посвящено множество работ (см., например, обзор [4]). 0001).

Работа выполнена в рамках государственного задания ИМ СО РАН (проект 0314-2019-

(c) 2020 Пожидаев А. П. 
Одним из главных примеров правосимметрических алгебр является следующий

Пример. Пусть $(\mathscr{A} ; \cdot)$ - коммутативная ассоциативная алгебра, а $D$ - ее дифференцирование. Тогда новое произведение $a \bullet b=D(a) \cdot b$ для всех $a, b \in \mathscr{A}$ превращает $\mathscr{A}$ в правосимметрическую алгебру.

Всюду далее $F$ обозначает основное поле, а $\mathscr{A}$ - ненулевую алгебру над полем $F$. Если не оговорено противное, для данного $A$ из алгебры матриц $M_{n}:=M_{n}(F)$ используется обозначение $A=\sum a_{i j} e_{i j}$, где $e_{i j}$ - обычные матричные единицы. Через $V_{n}$ обозначается $n$-мерное векторное пространство над $F$ со стандартным базисом строк $e_{i}, i \in \bar{n}:=\{1, \ldots, n\}$. Также используется обозначение $(x, y, z)_{r s}:=(x, y, z)-(x, z, y)$. Запись $\langle\Upsilon\rangle:=\langle\Upsilon\rangle_{F}$ используется для линейной оболочки множества $\Upsilon$ над $F$, где опускаем $F$, если поле ясно из контекста. Если $V$ - векторное пространство над $F$, то через $V^{*}$ обозначается дуальное пространство к $V$, а через $L(V)$ - алгебра всех $F$-линейных операторов на $V$.

\section{§ 1. Эндоморфы}

Рассмотрим прямую сумму алгебр $E(\mathscr{A}):=\mathscr{A} \oplus L(\mathscr{A})$ и наделим ее произведением по правилу:

$$
a \cdot A=a A, \quad A \cdot a=a A+\left[A, R_{a}\right]
$$

для всех $a \in \mathscr{A}, A \in L(\mathscr{A})$. Полученную алгебру назовем эндоморбом алгебры $\mathscr{A}$. По определению $\mathscr{A}$ и $L(\mathscr{A})$ являются подалгебрами в $E(\mathscr{A})$, а $\mathscr{A}$ - это правый модуль над $L(\mathscr{A})$.

ЗАмЕЧАНИЕ. Конструкция эндоморфа выглядит как конструкция голоморфа для алгебр Ли (см., например, [5]). Эта конструкция также является частным случаем общей конструкции расширения алгебры при помощи другой алгебры со взаимными действиями алгебр. Заметим, что все результаты данного параграфа практически дословно переносятся на случай супералгебр, при этом вторая формула в (3) преобразуется в

$$
A \cdot a=(-1)^{p(a) p(A)} a A+A R_{a}-(-1)^{p(a) p(A)} R_{a} A,
$$

где $p$ - функция четности. Ради простоты излагаем все для случая алгебр.

Предположим, что существует $\lambda \in \mathscr{A}^{*}$ такой, что $a b=\lambda(b) a$ (или $a b=$ $\lambda(a) b)$ для всех $a, b \in \mathscr{A}$. В этом случае говорим, что $\mathscr{A}$ - это алгебра скалярного умножения.

Теорема 1. Пусть $\mathscr{A}$ не является алгеброй скалярного умножения. Тогда алгебра $E(\mathscr{A})$ проста.

ДокАЗАТЕЛЬСтво. Пусть $I-$ собственный идеал в $E(\mathscr{A})$. По определению произведения в $E(\mathscr{A})$ справедливы следующие импликации:

$$
I \cap \mathscr{A} \neq 0 \Rightarrow \mathscr{A} \subseteq I ; I \cap L(\mathscr{A}) \neq 0 \Rightarrow L(\mathscr{A}) \subseteq I ; I \cap L(\mathscr{A}) \neq 0 \Rightarrow I \cap \mathscr{A} \neq 0 .
$$

Таким образом, если $I \cap \mathscr{A} \neq 0$, то $A \cdot a=a A+\left[A, R_{a}\right] \in I$ для всех $a \in \mathscr{A}, A \in$ $L(\mathscr{A})$. Поскольку $\mathscr{A}$ не является алгеброй скалярного умножения, существует $a \in \mathscr{A}$ такой, что $\left[A, R_{a}\right] \neq 0$ для некоторого $A \in L(\mathscr{A})$. Следовательно, $I \cap$ $L(\mathscr{A}) \neq 0$ и $I=E(\mathscr{A})$. Тем самым можно предполагать, что $I \cap \mathscr{A}=0$ и $I \cap L(\mathscr{A})=0$. 
Пусть $a+A \in I$ для некоторых ненулевых $a \in \mathscr{A}, A \in L(\mathscr{A})$. Тогда

$$
(a+A) \cdot B=a B+A B \in I, \quad B \cdot(a+A)=a B+\left[B, R_{a}\right]+B A \in I
$$

для любого $B \in L(\mathscr{A})$. Следовательно, $[A, B]-\left[B, R_{a}\right] \in I$ и $\left[R_{a}+A, B\right] \in$ $I \cap L(\mathscr{A})$ для каждого $B \in L(\mathscr{A})$. Значит, $R_{a}+A=\alpha E$ для некоторого $\alpha \in F$ ( $E$ обозначает тождественное отображение).

Далее, $b \cdot(a+A)=b \cdot\left(R_{a}+A\right)=\alpha b \in I$ для любого $b \in \mathscr{A}$, откуда $\alpha=0$. Таким образом,

$$
I=\left\{a-R_{a}: a \in \mathscr{A}\right\} .
$$

Так как $\left(a-R_{a}\right) \cdot A=a A-R_{a} A \in I$ для всех $a \in \mathscr{A}$ и $A \in L(\mathscr{A})$, то $R_{a A}=R_{a} A$, т. e.

$$
(x y) A=x(y A)
$$

для всех $x, y \in \mathscr{A}, A \in L(\mathscr{A})$. Полагая $A=R_{z}$ в (4), получаем, что $\mathscr{A}$ ассоциативна. Возьмем произвольный $y \in \mathscr{A}$. Предположим, что существует $x \in \mathscr{A}$ такой, что $x y \notin\langle y\rangle$. Выбирая $A$ в (4) так, что $y A=0$ и $(x y) A \neq 0$, приходим к противоречию. Таким образом, $x y=\alpha(x) y$ для некоторого $\alpha \in \mathscr{A}^{*}$ и $\mathscr{A}-$ алгебра скалярного умножения.

Положим $\mathscr{A}_{0}:=\mathscr{A}, \mathscr{A}_{k}=E\left(\mathscr{A}_{k-1}\right)$ при $k \geq 1$. Тогда теорема 1 дает критерий простоты алгебр $\mathscr{A}_{1}$ и получаем

Следствие 1. Алгебра $\mathscr{A}_{n}$ проста для всех $n>1$.

ДокАЗАтЕЛЬСтво. Достаточно заметить, что $E(\mathscr{A})$ неассоциативна и $E(\mathscr{A})$ не является алгеброй скалярного умножения для любой алгебры $\mathscr{A}$ такой, что $\operatorname{dim} \mathscr{A}>1$.

Теорема 2. Супералгебра $E(\mathscr{A})$ правосимметрическая тогда и только тогда, когда $\mathscr{A}$ - правосимметрическая супералгебра.

ДокАзАтЕльство. Докажем теорему для случая алгебр. В суперслучае доказательство получается аналогично или переходом к грассмановой оболочке.

Если $E(\mathscr{A})$ правосимметрическая, то $\mathscr{A}$ - правосимметрическая алгебра как подалгебра в $E(\mathscr{A})$. Предположим, что $\mathscr{A}$ правосимметрическая. Надо проверить тождество $(x, y, z)_{r s}=0$ в $E(\mathscr{A})$. В дальнейшем $A, B \in L(\mathscr{A})$ и $a, b \in \mathscr{A}$.

Рассмотрим $(A, a, b)_{r s}$. В этом случае

$$
\begin{aligned}
(A, a, b)=\left(a A+\left[A, R_{a}\right]\right) & \cdot b-(a b) A-\left[A, R_{a b}\right] \\
=a A b+b & \left.b A, R_{a}\right]+\left[\left[A, R_{a}\right], R_{b}\right]-(a b) A-\left[A, R_{a b}\right] \\
& =a A b+b A a-(b a) A+\left[\left[A, R_{a}\right], R_{b}\right]-(a b) A-\left[A, R_{a b}\right] .
\end{aligned}
$$

Меняя местами $a$ и $b$, получаем

$$
(A, a, b)_{r s}=\left[\left[A, R_{a}\right], R_{b}\right]-\left[A, R_{a b}\right]-\left[\left[A, R_{b}\right], R_{a}\right]+\left[A, R_{b a}\right] .
$$

Используя тождество Якоби, выводим

$$
(A, a, b)_{r s}=\left[\left[R_{b}, R_{a}\right], A\right]+\left[A, R_{[b, a]}\right] .
$$

Таким образом, $(A, a, b)_{r s}=0$ по (2).

Имеем $(a, A, b)_{r s}=0$, поскольку

$$
(a, A, b)=(a A) b-a(b A)-a\left[A, R_{b}\right]=(a b) A-a(b A), \quad(a, b, A)=(a b) A-a(b A) .
$$


Рассмотрим $(A, a, B)_{r s}$. В этом случае

$$
\begin{gathered}
(A, a, B)=\left(a A+\left[A, R_{a}\right]\right) B-A \cdot(a B)=a A B+\left[A, R_{a}\right] B-a B A-\left[A, R_{a B}\right] \\
\begin{array}{r}
(A, B, a)=a A B+\left[A B, R_{a}\right]-A \cdot\left(a B+\left[B, R_{a}\right]\right) \\
=a A B+\left[A B, R_{a}\right]-a B A-\left[A, R_{a B}\right]-A\left[B, R_{a}\right]
\end{array}
\end{gathered}
$$

откуда следует $(A, a, B)_{r s}=\left[A, R_{a}\right] B+A\left[B, R_{a}\right]-\left[A B, R_{a}\right]=0$, ввиду тождества $[x y, z]=[x, z] y+x[y, z]$, справедливого в ассоциативных алгебрах.

Окончательно $(a, A, B)_{r s}=0$, так как $(a, A, B)=(a A) B-a(A B)=0$.

\section{§ 2. Дифференцирования $\boldsymbol{E}(\mathscr{A})$ для унитальных $\mathscr{A}$}

Напомним, что линейное отображение $d \in L(\mathscr{A})$ называется дифферениированием алгебры $\mathscr{A}$, если $d(x y)=d(x) y+x d(y)$ для любых $x, y \in \mathscr{A}$. Множество всех дифференцирований алгебры $\mathscr{A}$ обозначается через $\operatorname{Der}(\mathscr{A})$. Если $d \in L(\mathscr{A})$, то отображение $D: L(\mathscr{A}) \mapsto L(\mathscr{A})$, определенное правилом $D(A)=[A, d]$, является внутренним дифференцированием алгебры $L(\mathscr{A})$. Как легко проверить, для любого $d \in \operatorname{Der}(\mathscr{A})$ отображение $\mathscr{D}: E(\mathscr{A}) \mapsto E(\mathscr{A})$ такое, что $(a+A) \mathscr{D}=a d+[A, d]$ для любых $a \in \mathscr{A}, A \in L(\mathscr{A})$, является дифференцированием $E(\mathscr{A})$. Обозначим такое дифференцирование $\mathscr{D}$ через $d+[\cdot, d]$. Следующая теорема говорит о том, что других дифференцирований на $E(\mathscr{A})$ не существует для унитальных алгебр $\mathscr{A}$.

Теорема 3. Пусть $\mathscr{A}$ - унитальная алгебра. Отображение $\mathscr{D}$ является дифференцированием $E(\mathscr{A})$ тогда и только тогда, когда $\mathscr{D}=d+[\cdot, d]$ для некоторого $d \in \operatorname{Der}(\mathscr{A})$.

ДоказАтельство. Пусть $\mathscr{D}$ - дифференцирование $E(\mathscr{A})$. Положим $x \mathscr{D}:=x_{\mathscr{D}}+x^{\mathscr{D}}$, где $x \in E(\mathscr{A}), x_{\mathscr{D}} \in \mathscr{A}, x^{\mathscr{D}} \in L(\mathscr{A})$. Очевидно, $0_{\mathscr{D}}=0^{\mathscr{D}}=0$. Возьмем $a, b \in \mathscr{A}$. Тогда по определению дифференцирования имеем

$$
\begin{aligned}
(a b) \mathscr{D}=(a b)_{\mathscr{D}}+(a b)^{\mathscr{D}}=\left(a_{\mathscr{D}}+a^{\mathscr{D}}\right) \cdot b+a \cdot\left(b_{\mathscr{D}}+b^{\mathscr{D}}\right) & \\
& =a_{\mathscr{D}} \cdot b+b \cdot a^{\mathscr{D}}+\left[a^{\mathscr{D}}, R_{b}\right]+a \cdot b_{\mathscr{D}}+a \cdot b^{\mathscr{D}},
\end{aligned}
$$

откуда

$$
\begin{gathered}
(a b)_{\mathscr{D}}=a_{\mathscr{D}} \cdot b+b \cdot a^{\mathscr{D}}+a \cdot b_{\mathscr{D}}+a \cdot b^{\mathscr{D}}, \\
(a b)^{\mathscr{D}}=\left[a^{\mathscr{D}}, R_{b}\right] .
\end{gathered}
$$

Пусть $1-$ единица в $\mathscr{A}$. Так как $R_{1}:=E-$ тождественное отображение, то $1^{\mathscr{D}}=0$ по (6). Полагая $a=1$ в $(6)$, получаем $b^{\mathscr{D}}=0$ для всех $b \in \mathscr{A}$. Следовательно, $\mathscr{D}$ инвариантно на $\mathscr{A}$ и его ограничение $d$ на $\mathscr{A}$ является дифференцированием $\mathscr{A}$ по (5).

Докажем, что $A_{\mathscr{D}}=0$ для всех $A \in L(\mathscr{A})$.

Для всех $A, B \in L(\mathscr{A})$ имеем

$$
\begin{gathered}
(A B) \mathscr{D}=\left(A_{\mathscr{D}}+A^{\mathscr{D}}\right) \cdot B+A \cdot\left(B_{\mathscr{D}}+B^{\mathscr{D}}\right), \\
(A B)_{\mathscr{D}}=A_{\mathscr{D}} \cdot B+B_{\mathscr{D}} \cdot A .
\end{gathered}
$$

Из (8) следует, что $[A, B]_{\mathscr{D}}=0$ для любых $A, B \in L(A)$. Так как $L(A)=$ $[L(A), L(A)]+\langle E\rangle$, а $E_{\mathscr{D}}=0$ по $(8)$, то $A_{\mathscr{D}}=0$ для всех $A \in L(\mathscr{A})$.

Значит, $\mathscr{D}$ инвариантно на $L(\mathscr{A})$ и его ограничение $D$ на $L(\mathscr{A})$ является дифференцированием $L(\mathscr{A})$ по (7). Следовательно, $D$ внутреннее согласно [6], 
и также обозначим через $D$ линейное отображение, для которого $A^{\mathscr{D}}=[A, D]$. Таким образом,

$$
(a+A) \mathscr{D}=a d+[A, D]
$$

для всех $a \in \mathscr{A}, A \in L(\mathscr{A})$.

Далее,

$$
(a A) \mathscr{D}=(a A) d=(a d) A+a[A, D]=(a d-a D) A+a A D .
$$

Подставляя $a=1$, замечаем, что $(1 D) A=0$ для всех таких $A \in L(\mathscr{A})$, что $1 A=0$. Это означает, что $1 D=\alpha 1$ для некоторого $\alpha \in F$. Кроме того,

$$
\left(a R_{b}\right) \mathscr{D}=(a b) d=(a d) R_{b}+a\left[R_{b}, D\right]
$$

для любых $a, b \in \mathscr{A}$. Подставляя $a=1$, получаем $b d=1\left[R_{b}, D\right]=b D-\alpha b$ для любого $b \in \mathscr{A}$. Следовательно, $D=d+\alpha E$. Так как $D$ определяется с точностью до скаляра, можно считать, что $D=d$. Таким образом, $\mathscr{D}=d+[\cdot, d]$.

Определим линейное отображение ${ }^{-}: V_{n} \mapsto M_{n}$ правилом $\bar{e}_{i}=e_{i i}$ (диагональное вложение). Наделим $V_{n}$ структурой алгебры, рассматривая $e_{i}$ как ортогональные идемпотенты для всех $i=1, \ldots, n$. Назовем $\mathscr{M}_{n}:=E\left(V_{n}\right)=V_{n} \oplus M_{n}$ правосимметрической алгеброй матричного типа (матричнал RS-алгебра, для краткости). Эта алгебра впервые возникла в [7] при классификации простых правосимметрических алгебр с унитальной матричной подалгеброй.

Теорема 4. Пусть $\mathscr{M}_{n}=V_{n} \oplus M_{n}$ - матричная RS-алгебра. Тогда ее алгебра дифференцирований тривиальна.

ДокАЗАтЕльство следует из теоремы 3, поскольку $V_{n}$ унитальна, $\operatorname{Der}\left(V_{n}\right)$ $=0$ и любое дифференцирование, определенное скаляром, нулевое.

\section{§ 3. Автоморфизмы матричных правосимметрических алгебр}

Напомним, что $\mathscr{M}_{n}:=V_{n} \oplus M_{n}$ имеет следующее произведение:

$$
(v+A)(u+B)=v \cdot u+v \cdot B+u \cdot A+[A, \bar{u}]+A B
$$

для всех $v, u \in V_{n}, A, B \in M_{n}$. В стандартном базисе имеем

$$
e_{i} \cdot e_{j}=\delta_{i j} e_{i}, e_{i j} \cdot e_{p q}=\delta_{j p} e_{i q}, \quad e_{i} \cdot e_{p q}=\delta_{i p} e_{q}, e_{p q} \cdot e_{i}=\delta_{i p} e_{q}+\delta_{i q} e_{p i}-\delta_{i p} e_{i q}
$$

для всех $i, j, p, q \in \bar{n}$, где $\delta$ - символ Кронекера. В дальнейшем обычно опускаем символ произведения · в $\mathscr{M}_{n}$.

Правые идеалы. Возьмем $S \subseteq \bar{n}$ и определим

$$
I_{S}:=\left\langle e_{k}-e_{s k}: s \in S, k \in \bar{n}\right\rangle \quad \mathscr{M}_{S}:=\left\langle e_{s k}: s \in S, k \in \bar{n}\right\rangle
$$

Предложение 1. Пусть $I \unlhd_{r} \mathscr{M}_{n}$. Тогда либо $I=V_{n} \oplus \mathscr{M}_{S}$, либо $I=I_{S}$ для некоторого $S \subseteq \bar{n}$.

ДокаЗАТЕЛЬСтво. Зафиксируем $S \subseteq \bar{n}$. Покажем, что $I_{S} \unlhd_{r} \mathscr{M}_{n}$.

Легко видеть, что $\left(e_{k}-e_{s k}\right) e_{j}=0$, если либо $k=s$, либо $j \notin\{s, k\}$. В противном случае $\left(e_{k}-e_{s k}\right) e_{j}= \pm\left(e_{k}-e_{s k}\right) \in I_{S}$. Более того, $\left(e_{k}-e_{s k}\right) e_{j r}=0$ при $j \neq k ;\left(e_{k}-e_{s k}\right) e_{k r}=e_{r}-e_{s r} \in I_{S}$, откуда $I_{S} \unlhd_{r} \mathscr{M}_{n}$.

Пусть $I-$ правый идеал в $\mathscr{M}_{n}$. Имеем два возможных случая.

СлУЧАЙ 1. $V_{n} \cap I \neq 0$. 
Легко видеть, что в этом случае $V_{n} \subseteq I$. Рассмотрим множество $J:=M_{n} \cap I$. Тогда $J \unlhd_{r} M_{n}$. Если $v+A \in I$ для некоторых $v \in V_{n}$ и $A \in M_{n}$, то $v \in I$ и $A \in I$, откуда $v+A \in V_{n} \oplus J$, т. е. $I \subseteq V_{n} \oplus J$. Из условия данного случая и выбора $J$ следует, что $V_{n} \oplus J \subseteq I$ и $I=V_{n} \oplus J$. Более того, если $A \in J$ и $a_{i j} \neq 0$, то имеем следующую цепочку включений:

$$
A e_{i} \in I \Rightarrow e_{i i} A \in I \Rightarrow e_{i i} A e_{j j} \in I \Rightarrow e_{i j} \in I \Rightarrow e_{i k} \in I \text { для всех } k \in \bar{n} .
$$

Следовательно, существует $S$ такое, что $J=\mathscr{M}_{S}$.

СлУчАй 2. $V_{n} \cap I=0$.

Рассмотрим два подмножества

$$
\begin{aligned}
& \mathscr{M}_{I}:=\left\{A \in M_{n}: v+A \in I \text { для некоторого } v \in V_{n}\right\} \\
& S:=\left\{i \in \bar{n}: a_{i j} \neq 0 \text { для некоторых } A \in \mathscr{M}_{I}, j \in \bar{n}\right\} .
\end{aligned}
$$

Покажем, что $I=I_{S}$.

Пусть $v+A \in I$, где $a_{i j} \neq 0$ для некоторых $i, j$. Тогда для некоторого $\alpha_{j} \in F$ имеем

$$
x:=v e_{j j}+A e_{j j}=\alpha_{j} e_{j}+A^{(j)} \in I \quad \text { при } A^{(j)}:=\sum_{p=1}^{n} a_{p j} e_{p j} .
$$

Если $i=j$, то $x e_{j}=\left(\alpha_{j}+a_{j j}\right) e_{j}+A^{(j)}-a_{j j} e_{j j} \in I$. Следовательно, $x-x e_{j}=-a_{j j} e_{j}+a_{j j} e_{j j} \in I$ и $e_{j}-e_{j j} \in I$.

Если $i \neq j$, то $x e_{i}=a_{i j} e_{j}-a_{i j} e_{i j} \in I$, т. е. $e_{j}-e_{i j} \in I$ в любом случае.

Далее, умножение справа на $e_{j k}$ дает $e_{k}-e_{i k} \in I$ для всех $k \in \bar{n}$. Таким образом, $I_{S} \subseteq I$.

Окончательно $v+A+\sum_{i, j} a_{i j}\left(e_{i}-e_{i j}\right) \in V_{n}$ и либо приходим к случаю 1 , либо $v+A \in I_{S}$, т. е. $I \subseteq I_{S}$ и $I=I_{S}$.

Положим

$$
I_{0}:=V_{n}, I_{k}:=\left\langle f_{i}^{(k)}:=e_{i}-e_{k i}: i \in \bar{n}\right\rangle, \quad k \in \bar{n} .
$$

Следствие 2. Правые идеалы $I_{0}, I_{1}, \ldots, I_{n}$ исчерпывают все минимальные ненулевые правые идеалы в $\mathscr{M}_{n}$.

Структура регулярного правого модуля. Рассмотрим $\mathscr{M}_{n}$ как правый $\mathscr{M}_{n}$-модуль, т. е. как правый регулярный модуль. Из следствия 2 видим, что $I_{0}, I_{1}, \ldots, I_{n}$ исчерпывают все неприводимые подмодули регулярного правого $\mathscr{M}_{n}$-модуля.

Лемма 1. Правые $\mathscr{M}_{n}$-модули $I_{k}$ и $I_{s}$ неизоморфны при $k \neq s$.

ДоКАЗАТЕЛЬСтво. Предположим, что $I_{0} \cong I_{k}$ для некоторого $k \in \bar{n}$. Если $\phi$ - изоморфизм $I_{0}$ и $I_{k}$, а $\phi\left(e_{k}\right)=\sum_{i=1}^{n} \alpha_{i} f_{i}^{(k)}$, то

$\phi\left(e_{k}\right)=\phi\left(e_{k} e_{k k}\right)=\phi\left(e_{k}\right) e_{k k}=\alpha_{k} f_{k}^{(k)} ; \phi\left(e_{k}\right)=\phi\left(e_{k} e_{k}\right)=\phi\left(e_{k}\right) e_{k}=-\sum_{i \neq k}^{n} \alpha_{i} f_{i}^{(k)} ;$

противоречие.

Предположим, что $I_{k} \cong I_{s}$ для некоторых $k \neq s$. Пусть $\phi-$ изоморфизм $I_{k}$ и $I_{s}$. Так как $\phi\left(f_{k}^{(k)}\right)=\phi\left(f_{k}^{(k)}\right) e_{k k}$ и $0=\phi\left(f_{k}^{(k)}\right) e_{k}$, то $\phi\left(f_{k}^{(k)}\right)=0$; противоречие.

Из предложения 1 и леммы 1 выводится 
Теорема 5. Регулярный правый $\mathscr{M}_{n}$-модуль является прямой суммой неприводимых неизоморфных правых $\mathscr{M}_{n}$-модулей $I_{k}: \mathscr{M}_{n}=I_{0} \oplus I_{1} \oplus \ldots \oplus I_{n}$. Любой правый регулярный подмодуль в $\mathscr{M}_{n}$ является прямой суммой неприводимых $\mathscr{M}_{n}$-модулей $I_{k}$, когда $k$ пробегает некоторое подмножество $S \subseteq \bar{n} \cup\{0\}$.

Ортогональные идемпотенты. Заметим, что $I_{0}$ обладает единственной (с точностью до перестановки) системой $\mathscr{E}:=\left\{e_{1}, \ldots, e_{n}\right\}$ из $n$ попарно ортогональных идемпотентов. Положим $f^{(k)}=-\sum_{i=1}^{n} f_{i}^{(k)}$. Тогда $\mathscr{F}_{k}:=\left\{f^{(k)}, f_{i}^{(k)}, i \in\right.$ $\bar{n} \backslash\{k\}\}$ - система ортогональных идемпотентов в $I_{k}$. Положим $e=-\sum_{i=1}^{n} e_{i}$.

Вычислим размерности левых аннуляторов этих элементов. Напомним, что левым аннулятором $\mathrm{Ann}_{l}(h)$ элемента $h$ называется множество

$$
\operatorname{Ann}_{l}(h):=\left\{x \in \mathscr{M}_{n}: x h=0\right\} .
$$

Имеем $e_{i j} e=-e_{j}, e_{i} e=-e_{i}$ и

$$
e_{i j} e_{k}=\left\{\begin{array}{ll}
e_{k} & \text { при } i=k, j=k, \\
e_{j}-e_{k j} & \text { при } i=k, j \neq k,
\end{array} \quad e_{i j} e_{k}= \begin{cases}e_{i k} & \text { при } i \neq k, j=k, \\
0 & \text { при } i \neq k, j \neq k .\end{cases}\right.
$$

Следовательно, $\operatorname{Ann}_{l}\left(e_{k}\right)=\left\{e_{i j}, e_{i}, e_{k}-e_{k k}: i \neq k, j \neq k\right\}$ и $\operatorname{Ann}_{l}(e)=\left\{e_{j j}-\right.$ $\left.e_{i j}, e_{i}-e_{i i}: i, j \in \bar{n}\right\}$, откуда $\operatorname{dim} \operatorname{Ann}_{l}\left(e_{k}\right)=n^{2}-n+1$ и $\operatorname{dim} \operatorname{Ann}_{l}(e)=n^{2}$.

Легко видеть, что $\operatorname{Ann}_{l}\left(e_{k}-e_{k k}\right)=\left\{e_{i j}: i \neq k\right\} \cup \mathscr{E}$ и $\operatorname{dim} \operatorname{Ann}_{l}\left(e_{k}-e_{k k}\right)=n^{2}$.

Рассмотрим идемпотенты $f_{i}^{(k)}, i \neq k$. Имеем следующие ненулевые произведения:

$$
\begin{gathered}
e_{i}\left(e_{i}-e_{k i}\right)=e_{i}, \quad e_{k}\left(e_{i}-e_{k i}\right)=-e_{i}, \\
e_{k j}\left(e_{i}-e_{k i}\right)= \begin{cases}-e_{k i} & \text { при } j=k, \\
e_{k i} & \text { при } j=i,\end{cases} \\
e_{i j}\left(e_{i}-e_{k i}\right)= \begin{cases}e_{i}, & \text { если } j=i, \\
e_{j}-e_{i j}, & \text { если } j \neq k, i,\end{cases} \\
e_{j k}\left(e_{i}-e_{k i}\right)= \begin{cases}e_{k}-e_{i k}-e_{i i} & \text { при } j=i, \\
-e_{j i} & \text { при } j \neq i,\end{cases} \\
e_{j i}\left(e_{i}-e_{k i}\right)=e_{j i}, \text { если } j \neq i, k .
\end{gathered}
$$

Отсюда получаем $\operatorname{dim} \operatorname{Ann}_{l}\left(e_{i}-e_{k i}\right)=n^{2}-n+1$ при $i \neq k$.

Лемма 2. $\operatorname{dim} \operatorname{Ann}_{l}\left(e_{k}\right)=n^{2}-n+1, \operatorname{dim}_{A^{\prime}}(e)=n^{2}, \operatorname{dim}_{l} \operatorname{Ann}_{l}\left(e_{k}-e_{k k}\right)=$ $n^{2}$ и $\operatorname{dim} \operatorname{Ann}_{l}\left(e_{i}-e_{k i}\right)=n^{2}-n+1$ для всех $i, k \in \bar{n}, i \neq k$.

Автоморфизмы $\mathscr{M}_{n}$. Пусть $\sigma-$ произвольная подстановка из $\mathbb{S}_{n}$. Как легко видеть, отображение $\phi_{\sigma}$ такое, что

$$
\phi_{\sigma}\left(e_{i}\right)=e_{\sigma(i)}, \quad \phi_{\sigma}\left(e_{i j}\right)=e_{\sigma(i) \sigma(j)},
$$

является автоморфизмом $\mathscr{M}_{n}$.

Рассмотрим множества ортогональных идемпотентов $\mathscr{E}$ и $\mathscr{F}_{k}$. Пусть $\phi-$ автоморфизм $\mathscr{M}_{n}$. Автоморфизмы переводят минимальные правые идеалы в такие же и отображают множества ортогональных идемпотентов в такие же, поэтому если $\phi\left(I_{0}\right)=I_{k}$, то $\phi(\mathscr{E})=\mathscr{F}_{k}$. По лемме $2 \phi(e)=f_{k}^{(k)}=e_{k}-e_{k k}$. Если $\phi\left(I_{k}\right)=I_{s}$ для некоторых $k, s \in \bar{n}$, то $\phi\left(e_{k}-e_{k k}\right)=e_{s}-e_{s s}$, что следует из свойств левых аннуляторов. 
Для доказательства следующей леммы удобно иметь таблицу умножения элементов из множеств $\mathscr{E}$ и $\mathscr{F}_{k}$ :

$$
\begin{gathered}
e_{i} \cdot e_{j}=\delta_{i j} e_{i}, \quad e_{i} \cdot f_{j}^{(p)}=\left(\delta_{i j}-\delta_{i p}\right) e_{j}, \quad f_{j}^{(p)} \cdot e_{i}=\delta_{i j} f_{i}^{(p)}-\delta_{i p} f_{j}^{(i)}, \\
f_{i}^{(p)} \cdot f_{j}^{(q)}=\left(\delta_{i j}-\delta_{p j}\right) f_{i}^{(p)}-\delta_{i q} f_{j}^{(p)} \\
e \cdot f_{j}^{(i)}=0=f_{j}^{(i)} \cdot e, \quad f^{(i)} \cdot f^{(j)}=f^{(i)}, \quad e \cdot e=-e ; \\
f^{(i)} \cdot f_{p}^{(j)}=-\delta_{i p} f^{(i)}, \quad f_{p}^{(j)} \cdot f^{(i)}=-\delta_{i p} f^{(j)} \\
f^{(i)} \cdot e_{j}=-f_{j}^{(i)}-\delta_{i j} f^{(i)}, \quad e_{j} \cdot f^{(i)}=-e_{j}-\delta_{i j} e
\end{gathered}
$$

для всех $i, j, p, q \in \bar{n}$.

Определим линейное отображение $\psi$ на $\mathscr{M}_{n}$ правилом

$$
\begin{gathered}
\psi\left(e_{1}\right)=f^{(1)}, \quad \psi\left(e_{i}\right)=f_{i}^{(1)}, \\
\psi\left(f_{1}^{(1)}\right)=e, \quad \psi\left(f_{i}^{(1)}\right)=e_{i}, \\
\psi\left(f_{1}^{(k)}\right)=f^{(k)}, \quad \psi\left(f_{i}^{(k)}\right)=f_{i}^{(k)}
\end{gathered}
$$

для всех $i, k \geq 2$.

Лемма 3. Отображение $\psi$ является автоморфизмом $\mathscr{M}_{n}$.

ДокАЗАТЕЛЬСтво проводится непосредственной проверкой. Требуется доказать, что

$$
\psi\left(f_{i}^{(p)} \cdot f_{j}^{(q)}\right)=\psi\left(f_{i}^{(p)}\right) \cdot \psi\left(f_{j}^{(q)}\right)
$$

для всех $i, j \in \bar{n}, p, q \in \bar{n} \cup\{0\}$, где $f_{i}^{(0)}:=e_{i}$. Мы рассматриваем отдельно случаи, когда по крайней мере один индекс в (9) равен 0 или 1 . Полностью разберем случай $p=0$ и $i \geq 2$ :

$$
\begin{gathered}
\psi\left(e_{i} \cdot f_{1}^{(0)}\right)=0=f_{i}^{(1)} \cdot f^{(1)}=\psi\left(e_{i}\right) \cdot \psi\left(f_{1}^{(0)}\right) \\
\psi\left(e_{i} \cdot f_{p}^{(0)}\right)=\delta_{i p} \psi\left(e_{i}\right)=\delta_{i p} f_{i}^{(1)}=\psi\left(e_{i}\right) \cdot \psi\left(f_{p}^{(0)}\right) ; \\
\psi\left(e_{i} \cdot f_{1}^{(1)}\right)=0=f_{i}^{(1)} \cdot e=\psi\left(e_{i}\right) \cdot \psi\left(f_{1}^{(1)}\right) ; \\
\psi\left(e_{i} \cdot f_{p}^{(1)}\right)=\psi\left(\delta_{i p} e_{p}\right)=\delta_{i p} f_{p}^{(1)}=f_{i}^{(1)} \cdot e_{p}=\psi\left(e_{i}\right) \cdot \psi\left(f_{p}^{(1)}\right) ; \\
\psi\left(e_{i} \cdot f_{1}^{(j)}\right)=\psi\left(-\delta_{i j} e_{1}\right)=-\delta_{i j} f^{(1)}=f_{i}^{(1)} \cdot f^{(j)}=\psi\left(e_{i}\right) \cdot \psi\left(f_{1}^{(j)}\right) ; \\
\psi\left(e_{i} \cdot f_{p}^{(j)}\right)=\psi\left(\left(\delta_{i p}-\delta_{i j}\right) e_{p}\right)=\left(\delta_{i p}-\delta_{i j}\right) f_{p}^{(1)}=f_{i}^{(1)} \cdot f_{p}^{(j)}=\psi\left(e_{i}\right) \cdot \psi\left(f_{p}^{(j)}\right) .
\end{gathered}
$$

Из оставшихся случаев рассмотрим, например, следующий (при $i, j, k \geq 2$ ):

$$
\begin{gathered}
\psi\left(f_{1}^{(i)} \cdot f_{j}^{(1)}\right)=\psi\left(-\delta_{i j} f_{1}^{(i)}-f_{j}^{(i)}\right)=-\delta_{i j} f^{(i)}-f_{j}^{(i)}, \\
\psi\left(f_{1}^{(i)}\right) \cdot \psi\left(f_{j}^{(1)}\right)=f^{(i)} \cdot e_{j}=-f_{j}^{(i)}-\delta_{i j} f^{(i)} .
\end{gathered}
$$

Остальные случаи рассматриваются аналогично. Лемма доказана. 
Предложение 2. Пусть $\phi \in \operatorname{Aut}\left(\mathscr{M}_{n}\right), \phi\left(I_{0}\right)=I_{0}$, и $\phi\left(I_{p}\right)=I_{\sigma(p)}$ для всех $p \in \bar{n}$ и некоторого $\sigma \in \mathbb{S}_{n}$. Тогда $\phi=\phi_{\sigma} . B$ частности, если $\phi\left(I_{k}\right)=I_{k}$ для всех $k \in \bar{n}$, то $\phi=1$.

ДокАзАтЕльство. Мы должны показать, что $\phi\left(e_{t}\right)=e_{\sigma(t)}, \phi\left(e_{t p}\right)=e_{\sigma(t) \sigma(p)}$ для всех $t, p \in \bar{n}$. Рассмотрим произвольный $e_{p}-e_{t p} \in I_{t}, p \neq t$. Пусть $s:=\sigma(t)$, $\phi\left(e_{t}\right):=e_{i}, \phi\left(e_{p}\right):=e_{j}$ при $i \neq j$ и $\phi\left(e_{p}-e_{t p}\right):=e_{k}-e_{s k}$ при $k \neq s$ и некоторых $i, j, k \in \bar{n}$. Так как $\phi\left(e_{t}-e_{t t}\right)=e_{s}-e_{s s}$, то $\phi\left(e_{t p}\right)=e_{j}-e_{k}+e_{s k}$, $\phi\left(e_{t t}\right)=e_{i}-e_{s}+e_{s s}$. Тогда

$$
\phi\left(e_{t p}\right)=\phi\left(e_{t t} e_{t p}\right)=\left(e_{i}-e_{s}+e_{s s}\right)\left(e_{j}-e_{k}+e_{s k}\right)=\left(\delta_{i s}-\delta_{i k}-1\right) e_{k}+e_{s k},
$$

откуда $j=k, i=s$. Таким образом,

$$
\phi\left(e_{t p}\right)=e_{\sigma(t) k}, \quad \phi\left(e_{t}\right)=e_{\sigma(t)}, \quad \phi\left(e_{t t}\right)=e_{\sigma(t) \sigma(t)}
$$

для некоторого $k \neq \sigma(t)\left(k:=k_{p}\right.$ зависит от $\left.p\right)$. Следовательно,

$$
e_{\sigma(t) \sigma(t)}=\phi\left(e_{t t}\right)=\phi\left(e_{t p} e_{p t}\right)=e_{\sigma(t) k_{p}} \cdot e_{\sigma(p) k_{t}},
$$

откуда $\sigma(p)=k_{p}, \sigma(t)=k_{t}$, т. е. $\phi\left(e_{t p}\right)=e_{\sigma(t) \sigma(p)}$. Окончательно наше утверждение следует из произвольности выбора $p$ и $t(p \neq t)$.

Теорема 6. Пусть $\phi \in \operatorname{Aut}\left(\mathscr{M}_{n}\right)$. Тогда либо $\phi=\phi_{\sigma}$, либо $\phi=\phi_{\sigma} \circ \psi \circ \phi_{\tau}$ для некоторых $\sigma, \tau \in \mathbb{S}_{n}$. Кроме того, $\phi\left(M_{n}\right)=M_{n}$.

ДокАЗАтЕЛЬСтво. Рассмотрим произвольный $\phi \in \operatorname{Aut}\left(\mathscr{M}_{n}\right)$. Предположим, что $\phi\left(I_{0}\right)=I_{k}$ для некоторого $k \in \bar{n}$. Заметим, что $\theta:=\psi \circ \phi_{\tau}$ отображает $I_{0}$ на $I_{k}$, где $\tau \in \mathbb{S}_{n}$ выбран так, что $\tau(1)=k$. Тогда $\theta^{-1}\left(I_{k}\right)=I_{0}$. Следовательно, $\phi \circ \theta^{-1}\left(I_{0}\right)=I_{0}$ и $\phi \circ \theta^{-1}=\phi_{\sigma}$ для некоторого $\sigma \in \mathbb{S}_{n}$ по предложению 2 . Значит, $\phi=\phi_{\sigma} \circ \theta=\phi_{\sigma} \circ \psi \circ \phi_{\tau}$.

Чтобы доказать последнее утверждение, достаточно заметить, что по определению $\psi$ имеем

$$
\psi\left(e_{j k}\right)=e_{j k}-e_{1 k}, \quad \psi\left(e_{1 j}\right)=-e_{1 j}, \quad \psi\left(e_{i 1}\right)=\sum_{t=1}^{n}\left(e_{1 t}-e_{i t}\right) \in M_{n}
$$

для всех $i, j, k \in \bar{n}, k, j \neq 1$. Таким образом, $\psi\left(M_{n}\right)=M_{n}$. По определению $\phi_{\sigma}$ имеем $\phi_{\sigma}\left(M_{n}\right)=M_{n}$. Следовательно, $\phi\left(M_{n}\right)=M_{n}$.

Лемма 4. $\phi_{\tau_{1}} \circ \psi \circ \phi_{\sigma_{1}}=\phi_{\tau_{2}} \circ \psi \circ \phi_{\sigma_{2}} \Longleftrightarrow \sigma_{1}(1)=\sigma_{2}(1), \tau_{1} \sigma_{1}=\tau_{2} \sigma_{2}$.

ДокАзАтЕЛЬство. Покажем, что $\psi=\phi_{\tau} \circ \psi \circ \phi_{\sigma} \Longleftrightarrow \sigma(1)=\tau(1)=1$, $\tau=\sigma^{-1}$.

Действительно, так как $\psi\left(I_{0}\right)=I_{1}, \psi\left(I_{1}\right)=I_{0}, \psi\left(I_{k}\right)=I_{k}$ для всех $k \geq 2$, то $\psi=\phi_{\tau} \circ \psi \circ \phi_{\sigma}$ влечет $\sigma(1)=\tau(1)=1, \sigma(\tau(k))=k$, т. е. $\tau=\sigma^{-1}$. Обратно, если $\sigma(1)=\tau(1)=1$ и $\tau=\sigma^{-1}$, то $\theta:=\phi_{\tau} \circ \psi \circ \phi_{\sigma}$ действует на множестве минимальных правых идеалов следующим образом: $\theta\left(I_{0}\right)=I_{1}, \theta\left(I_{1}\right)=I_{0}, \theta\left(I_{k}\right)=I_{k}$ для всех $k \geq 2$. Значит, $\left(\theta^{-1} \circ \psi\right)\left(I_{i}\right)=I_{i}$ для всех $i=0, \ldots, n$, откуда $\theta^{-1} \circ \psi=1$ по предложению 2. Кроме того,

$$
\phi_{\tau_{1}} \circ \psi \circ \phi_{\sigma_{1}}=\phi_{\tau_{2}} \circ \psi \circ \phi_{\sigma_{2}} \Longleftrightarrow \psi=\phi_{\tau_{1}^{-1} \tau_{2}} \circ \psi \circ \phi_{\sigma_{2} \sigma_{1}^{-1}},
$$

что эквивалентно $\tau_{1} \sigma_{1}=\tau_{2} \sigma_{2}, \sigma_{1}(1)=\sigma_{2}(1)$. 
Теорема 7. $\operatorname{Aut}\left(\mathscr{M}_{n}\right) \cong \mathbb{S}_{n+1}$.

ДокАЗАТЕЛЬСТво. По предложению 2, теореме 6 и лемме $4\left|\operatorname{Aut}\left(\mathscr{M}_{n}\right)\right|=$ $(n+1)$ ! Так как автоморфизмы $\psi$ и $\phi_{\sigma}\left(\sigma \in \mathbb{S}_{n}\right)$ порождают $\operatorname{Aut}\left(\mathscr{M}_{n}\right)$ и дают все перестановки между $I_{k}$ и $I_{s}$ для всех $k, s=0,1, \ldots, n$, суперпозиции автоморфизмов $\psi$ и $\phi_{\sigma}$ дают все возможные перестановки минимальных правых идеалов. По предложению 2 любая такая перестановка однозначно определяет автоморфизм из $\operatorname{Aut}\left(\mathscr{M}_{n}\right)$.

Благодарность. Результаты данной статьи были доложены на семинаре по теории колец им. А. И. Ширшова (ИМ СО РАН, 12.02.2020). Автор благодарен рецензенту за множество ценных замечаний, позволивших улучшить изложение статьи.

\section{ЛИТЕРАТУРА}

1. Koszul J.-L. Domaines bornés homogènes et orbites de groupes de transformations affines // Bull. Soc. Math. France. 1961. V. 89. P. 515-533.

2. Vinberg E. B. The theory of convex homogeneous cones // Trans. Moscow Math. Soc. 1963. V. 12. P. 1033-1047.

3. Gerstenhaber M. On the deformation of rings and algebras // Ann. Math. Second Series. 1964. V. 79, N 1. P. 59-103.

4. Burde D. Left-symmetric algebras, or pre-Lie algebras in geometry and physics // Centr. Eur. J. Math. 2006. V. 4. P. 323-357.

5. Bourbaki N. Lie groups and Lie algebras. Paris: Hermann Reading; Mass.: Addison-Wesley, 1975.

6. Słowik R. Derivations of rings of infinite matrices // Comm. Algebra. 2015. V. 43, N 8. P. 3433-3441.

7. Pozhidaev A. P., Shestakov I. P. The right-symmetric algebras possessing a "unital" matrix subalgebra // Mal'tsev meeting, Novosibirsk, 2019. 180.

Поступила в редакиию 18 марта 2020 г.

После доработки 19 мая 2020 г.

Принята к публикации 17 июня 2020 2.

Пожидаев Александр Петрович Институт математики им. С. Л. Соболева СО РАН, пр. Академика Коптюга, 4, Новосибирск 630090; Новосибирский государственный университет, ул. Пирогова, 1, Новосибирск 630090 app@math.nsc.ru 